\title{
TOPOGRAPHIC CHANGES ON AIRLESS BODIES: \\ THEORETICAL CONSIDERATIONS
}

(Additions to the article by L. B. Ronca and R. B. Furlong, The Moon 17, 233-237)

(Received 3 April, 19978)

In a presentation at the Ninth Lunar and Planetary Science Conference (Gault and Wedekind, 1978) it was shown that "the differential probability $\mathrm{d} P$ for an object to impact a spherical body at an angle $\theta$ is $\mathrm{d} P=2 \sin \theta \cos \theta \mathrm{d} \theta$ ". This relationship can be made to apply to one of the assumptions of Ronca and Furlong's paper. The cumulative probability of impacts at angle from $0^{\circ}$ to $\theta$ will be

$$
P_{\text {cum }}=\int_{0}^{\theta} 2 \sin \theta \cos \theta \mathrm{d} \theta=\sin ^{2} \theta
$$

Within the ball-park character of the calculation, the $\sin ^{2} \theta$ does not differ significantly from the linear relationship assumed by Equation (8), p. 237, of the above paper.

\section{Reference}

Gault, D. E. and Wedekind, J. A.: 1978, 'Experimental Studies of Oblique Impacts' (abstract), Ninth Lunar and Planetary Science Conference, Part 1, pp. 374-376. 